Bull. Korean Math. Soc. 46 (2009), No. 2, pp. 289-294

DOI 10.4134/BKMS.2009.46.2.289

\title{
ON EINSTEIN HERMITIAN MANIFOLDS II
}

\author{
JAEMAN KIM
}

\begin{abstract}
We show that on a Hermitian surface $M$, if $M$ is weakly *Einstein and has $J$-invariant Ricci tensor then $M$ is Einstein, and vice versa. As a consequence, we obtain that a compact $*$-Einstein Hermitian surface with $J$-invariant Ricci tensor is Kähler. In contrast with the 4dimensional case, we show that there exists a compact Einstein Hermitian $(4 n+2)$-dimensional manifold which is not weakly $*$-Einstein.
\end{abstract}

\section{Introduction}

The Riemannian version of the Goldberg-Sachs Theorem [1] says that the self-dual Weyl tensor $W^{+}$of an Einstein Hermitian surface $M$ is degenerate, i.e., at least two of its three eigenvalues coincide. In fact, this implies that $M$ is weakly $*$-Einstein. Another weak form of the Einstein condition is the $J$-invariant condition for the Ricci tensor. In this note, we show that, on a Hermitian surface, both weak versions of the Einstein condition together are equivalent to the Einstein condition. In contrast with the 4-dimensional case, we show that there exists a compact Einstein Hermitian $(4 n+2)$-dimensional manifold which is not weakly $*$-Einstein. More precisely we obtain the followings:

Theorem 1. Let $M=(M, J, g)$ be a Hermitian surface. If $M$ is weakly *Einstein and has $J$-invariant Ricci tensor, then $M$ is Einstein, and vice versa.

Corollary 1. A compact $*$-Einstein Hermitian surface with J-invariant Ricci tensor is Kähler.

Theorem 2. If $M=\left(S^{2 n+1} \times S^{2 n+1}, J_{C}, g_{\text {prod }}\right)$, then $M$ is a compact Einstein Hermitian manifold which is not weakly $*$-Einstein, where $J_{C}$ is the complex structure of Calabi-Eckmann and $g_{\text {prod }}$ the standard product metric on the product of the spheres.

Received May 13, 2008.

2000 Mathematics Subject Classification. 53A30, 53B35, 53C25, 53C55, 53C56.

Key words and phrases. Hermitian surface, weakly *-Einstein, J-invariant Ricci tensor, Einstein, vice versa, *-Einstein, Kähler, compact Einstein Hermitian $(4 n+2)$-dimensional manifold.

This study is supported by Kangwon National University. 
We recall that a Hermitian manifold $(M, J, g)$ is called Einstein Hermitian if the compatible Riemannian metric $g$ with complex structure $J$ is Einstein by its Levi-Civita connection.

\section{Preliminaries}

Let $M=(M, J, g)$ be a Hermitian manifold with complex structure $J$ and compatible Riemannian metric $g$, i.e., $g(J X, J Y)=g(X, Y)$ for vector fields $X, Y$. Denote by $\Omega(X, Y)$ the Kähler form of $M$ defined by $\Omega(X, Y)=$ $g(J X, Y)$. We shall always consider $M$ with the orientation determined by the complex structure $J$. The Riemannian curvature $R$, the Ricci tensor Ric and the scalar curvature $s$ of $M$ are defined by

$$
\begin{gathered}
R(X, Y) Z=\left[\nabla_{X}, \nabla_{Y}\right] Z-\nabla_{[X, Y]} Z, \\
\operatorname{Ric}(X, Y)=\operatorname{Trace}\{Z \rightarrow R(Z, X) Y\}, s=\text { Trace }_{g} \operatorname{Ric}
\end{gathered}
$$

for vector fields $X, Y, Z$.

Furthermore, we define the $*$-Ricci tensor and $*$-scalar curvature of $(J, g)$ by

$$
\operatorname{Ric}^{*}(X, Y)=\operatorname{Trace}\{Z \rightarrow-J R(Z, X) J Y\}, s^{*}=\operatorname{Trace}_{g} \operatorname{Ric}^{*} .
$$

The $*$-Ricci tensor is in general neither symmetric nor skew-symmetric and satisfies the equation: $\operatorname{Ric}^{*}(J X, J Y)=\operatorname{Ric}^{*}(Y, X)$. Note that on a Kähler manifold, the $*$-Ricci tensor and the Ricci tensor coincide; this is a consequence of the Kähler identity $R(X, Y)(J Z)=J(R(X, Y) Z)$, which itself follows from the fact that $\nabla J=0$. We shall say that $M$ is weakly $*$-Einstein if and only if the $*$-Ricci tensor is a functional multiple of the metric $g$, i.e., Ric $^{*}=\lambda g$. Note that in contrast to Einstein manifolds the $*$-scalar curvature of a weakly *-Einstein manifold $M$ need not be a constant and when this holds, we shall simply say that $M$ is *-Einstein. We also consider the curvature tensor $R$ as a $(0,4)$-tensor as follows: $R(X, Y, Z, W)=-g(R(X, Y) Z, W)$. Considering the Riemannian curvature tensor $R$ as a $(0,4)$-tensor, we have the following well known $S O(2 n)$-decomposition: $R=\frac{s}{4 n(2 n-1)} g \wedge g+\frac{1}{2 n-2} \operatorname{Ric}_{0} \wedge g+W$, where $\operatorname{Ric}_{0}=\operatorname{Ric}-\frac{s}{2 n} g$ is the traceless Ricci tensor and $W$ is the Weyl tensor. Here the symbol $\wedge$ is the Nomizu-Kulkarni product of symmetric $(0,2)$-tensors generating a curvature type tensor. Note that $\operatorname{Ric}_{0}=0$ if and only if $M$ is Einstein. Let $\left\{e_{i}\right\}_{i=1, \ldots, 2 n}$ be a local orthonormal frame and $R_{i j k l}, r_{i j}, R_{i j}$, $W_{i j k l}, r_{i j}^{*}, R_{i j}^{*}$ be components of $R, \mathrm{Ric}, \mathrm{Ric}_{0}, W, \mathrm{Ric}^{*}, \mathrm{Ric}_{0}^{*}$ with respect to $\left\{e_{i}\right\}$ respectively, i.e.,

$$
\begin{gathered}
R_{i j k l}=R\left(e_{i}, e_{j}, e_{k}, e_{l}\right), r_{i j}=\operatorname{Ric}\left(e_{i}, e_{j}\right), R_{i j}=\operatorname{Ric}_{0}\left(e_{i}, e_{j}\right), \\
W_{i j k l}=W\left(e_{i}, e_{j}, e_{k}, e_{l}\right), r_{i j}^{*}=\operatorname{Ric}^{*}\left(e_{i}, e_{j}\right), R_{i j}^{*}=\operatorname{Ric}_{0}^{*}\left(e_{i}, e_{j}\right) .
\end{gathered}
$$

Here $\mathrm{Ric}_{0}^{*}$ is the traceless $*$-Ricci tensor. Hence the Weyl tensor $W=\left(W_{i j k l}\right)$ of $M$ can be expressed as

$$
W_{i j k l}=R_{i j k l}-\frac{1}{2 n-2}\left(R_{i k} \delta_{j l}+R_{j l} \delta_{i k}-R_{i l} \delta_{j k}-R_{j k} \delta_{i l}\right)-
$$




$$
-\frac{s}{2 n(2 n-1)}\left(\delta_{i k} \delta_{j l}-\delta_{i l} \delta_{j k}\right)
$$

Here $\delta_{i j}$ is the Kronecker delta. We denote $J_{i j}$ by

$$
J_{i j}=g\left(J e_{i}, e_{j}\right)
$$

From now on the components of tensors shall be considered under orthonormal frame. Indices with an overbar are the ones with respect to $\left\{J e_{i}\right\}$, for example,

$$
R_{\bar{i} j k l}=R\left(J e_{i}, e_{j}, e_{k}, e_{l}\right) .
$$

Using this notation, we have

$$
r_{i j}=\sum_{a} R_{i a j a}, r_{i j}^{*}=\sum_{a} R_{i a \bar{j} \bar{a}}, s=\sum_{a, b} R_{a b a b}, s^{*}=\sum_{a, b} R_{a b \bar{a} \bar{b}}
$$

and

$$
r_{i j}^{*}=r_{j i}^{*} .
$$

The Riemannian metric $g$ induces a metric on the bundle $\bigwedge^{2} M$ of 2-vectors on $M$ by $\left\langle X_{1} \wedge X_{2}, X_{3} \wedge X_{4}\right\rangle=\operatorname{det}\left(g\left(X_{i}, X_{j}\right)\right)$. Then we also consider the curvature tensor $R$ as an endomorphism of the bundle $\wedge^{2} M$ as follows: $\langle R(X \wedge Y), Z \wedge$ $W\rangle=-g(R(X, Y) Z, W)$. In dimension 4, the Hodge star operator defines an endomorphism $*$ of $\bigwedge^{2} M$ with $*^{2}=I d$. Hence $\bigwedge^{2} M=\bigwedge^{+} M \bigoplus \bigwedge^{-} M$, where $\wedge^{+} M\left(\right.$ resp. $\left.\wedge^{-} M\right)$ is the subbundle of $\wedge^{2} M$ corresponding to the eigenvalue +1 (resp. -1 ) of $*$. As an endomorphism of $\bigwedge^{2} M$ the Weyl tensor $W$ commutes with the Hodge star operator $*$ and so $W$ preserves the decomposition $\wedge^{2} M=$ $\wedge^{+} M \bigoplus \wedge^{-} M$. Note that as an endomorphism of $\bigwedge^{2} M$ the curvature tensor $R$ commutes with the Hodge star operator $*$, i.e., $* R=R *$ if and only if $M$ is Einstein [5]. We denote the restriction of $W$ to $\bigwedge^{+} M\left(\operatorname{resp} . \bigwedge^{-} M\right)$ by $W^{+}$(resp. $W^{-}$) called the self-dual Weyl tensor (resp. anti-self-dual Weyl tensor). Hence, in dimension 4, the Riemannian curvature $(0,4)$-tensor $R$ can be obtained as follows:

$$
R=\frac{s}{24} g \wedge g+\frac{1}{2} \operatorname{Ric}_{0} \wedge g+W^{+}+W^{-} .
$$

Let $M=(M, J, g)$ be a Hermitian surface (i.e., a Hermitian manifold of real dimension 4 ). From now on we identify 2 -vectors with 2 -forms. Then usual type decomposition

$$
\bigwedge^{2} M \bigotimes C=\bigwedge^{2,0} M \bigoplus \bigwedge^{1,1} M \bigoplus \bigwedge^{0,2} M
$$

of complexified 2-forms induces the decomposition

$$
\bigwedge^{2} M=R \Omega \bigoplus\left(\bigwedge_{0}^{1,1} M\right)_{R} \bigoplus\left(\bigwedge^{2,0} M \bigoplus \bigwedge^{0,2} M\right)_{R}
$$


where $R \Omega$ is the line bundle generated by the Kähler form $\Omega$ and $\bigwedge_{0}^{1,1} M$ is the orthogonal complement of $R \Omega$ in $\bigwedge^{1,1} M$. Note that

$$
R \Omega \bigoplus\left(\bigwedge^{2,0} M \bigoplus \bigwedge^{0,2} M\right)_{R}=\bigwedge^{+} M
$$

and

$$
\left(\bigwedge_{0}^{1,1} M\right)_{R}=\bar{\bigwedge} M
$$

On the other hand we can extend $J$ to act on 2-forms as follows: $J(A)(X, Y)=$ $A(J X, J Y)$ for a 2 -form $A$.

\section{Proof of Theorem 1 and Corollary 1}

Let $M=(M, J, g)$ be a Hermitian surface. From now on we assume that all tensors are continued by complex linearity. For an orthonormal frame $\left\{e_{1}, J e_{1}, e_{2}, J e_{2}\right\}$ we set $Z_{k}=\frac{1}{\sqrt{2}}\left(e_{k}-i J e_{k}\right), Z_{\bar{k}}=\frac{1}{\sqrt{2}}\left(e_{k}+i J e_{k}\right), k=1,2$. Let $\langle$,$\rangle be the Hermitian continuation of g$ on $\bigwedge^{2} M \otimes C$ and $\alpha=\left(Z_{1} \wedge Z_{2}\right), \beta=$ $\frac{1}{\sqrt{2}}\left(Z_{1} \bigwedge Z_{\overline{1}}+Z_{2} \bigwedge Z_{\overline{2}}\right), \bar{\alpha}=\left(Z_{\overline{1}} \bigwedge Z_{\overline{2}}\right), \gamma=\left(Z_{1} \bigwedge Z_{\overline{2}}\right), \delta=\frac{1}{\sqrt{2}}\left(Z_{1} \bigwedge Z_{\overline{1}}-\right.$ $\left.Z_{2} \wedge Z_{\overline{2}}\right), \bar{\gamma}=\left(Z_{\overline{1}} \wedge Z_{2}\right)$. Then $\{\alpha, \beta, \bar{\alpha}\}$ and $\{\gamma, \delta, \bar{\gamma}\}$ are orthonormal frames of $\bigwedge^{+} M \otimes C$ and $\Lambda^{-} M \otimes C$ respectively. Consider $W^{+}$as an endomorphism of $\bigwedge^{+} M \otimes C$. Then the matrix of $W^{+}$with respect to the frame $\{\alpha, \beta, \bar{\alpha}\}$ has the following form [2]:

$$
W^{+}=\left(\begin{array}{c|c|c}
W_{1}^{+} & W_{2}^{+} & W_{3}^{+} \\
\hline W_{2}^{+} & -2 W_{1}^{+} & -W_{2}^{+} \\
\hline W_{3}^{+} & -W_{2}^{+} & W_{1}^{+}
\end{array}\right) .
$$

Here $W_{1}^{+}=\langle R \alpha, \alpha\rangle-\frac{s}{12}, W_{2}^{+}=\langle R \alpha, \beta\rangle, W_{3}^{+}=\langle R \alpha, \bar{\alpha}\rangle$. Since $\nabla_{X} Y \in T^{(1,0)} M$ for all $X, Y \in T^{(1,0)} M$, we have $W_{3}^{+}=0$. Hence, on a Hermitian surface $M$, $W^{+}$is degenerate if and only if $W_{2}^{+}=0$. In order to show that Theorem 1 and Corollary 1 hold, we need the following;

Lemma 1. Let $M=(M, J, g)$ be a Hermitian surface. Then we have

(2) $\operatorname{Ric}(X, Y)+\operatorname{Ric}(J X, J Y)-\operatorname{Ric}^{*}(X, Y)-\operatorname{Ric}^{*}(J X, J Y)=\frac{s-s^{*}}{2} g(X, Y)$.

Proof. Let $\left\{e_{i}\right\}_{i=1, \ldots, 4}$ be an orthonormal frame and $\left\{e^{i}\right\}_{i=1, \ldots, 4}$ its dual frame. Then we can write the Kähler form $\Omega$ as $\Omega=\frac{1}{2} \sum J_{i j} e^{i} \wedge e^{j}$ and we have

$$
W(\Omega)_{k l}=\frac{1}{2} \sum_{i, j} W_{i j k l} J_{i j}=\frac{1}{2} \sum_{i} W_{\bar{i} \bar{i} k l}=\sum_{i} W_{i k \bar{i} l} .
$$


From (1), we have

$$
\begin{aligned}
W(\Omega)_{k l} & =-r_{k \bar{l}}^{*}-\frac{1}{2}\left(R_{\bar{k} l}-R_{k \bar{l}}\right)-\frac{s}{12} J_{k l} \\
& =-r_{(k \bar{l})}^{*}-r_{[k \bar{l}]}^{*}-\frac{1}{2}\left(R_{\bar{k} l}+R_{\overline{\bar{k}} \bar{l}}\right)-\frac{s}{12} J_{k l} \\
& =R_{(\bar{k} l)}^{*}+\frac{s^{*}}{4} J_{k l}-r_{[\bar{k} l]}^{*}-\frac{1}{2}\left(R_{\bar{k} l}+R_{\overline{\bar{k}} \bar{l}}\right)-\frac{s}{12} J_{k l},
\end{aligned}
$$

where $A_{(i j)}$ and $A_{[i j]}$ are the symmetric and skew-symmetric part of a tensor $A_{i j}$, respectively. It is easy to see that only $B_{k l}=R_{(\bar{k} l)}^{*}-\frac{1}{2}\left(R_{\bar{k} l}+R_{\overline{\bar{k}} \bar{l}}\right)$ is the component of a section of $\left(\bigwedge_{0}^{1,1} M\right)_{R}$. Since the Weyl tensor of $M$ preserves the self-duality and $\left(\bigwedge_{0}^{1,1} M\right)_{R}$ is identified with $\bigwedge^{-} M$, we get $B_{k l}=0$. Therefore the identity (2) holds. This completes the proof of Lemma 1.

Suppose that a Hermitian surface $M$ is weakly $*$-Einstein. Then the above identity (2) implies $\operatorname{Ric}(X, Y)+\operatorname{Ric}(J X, J Y)=\frac{s}{2} g(X, Y)$. By the another assumption, i.e., $M$ has $J$-invariant Ricci tensor, we get $\operatorname{Ric}(X, Y)=\frac{s}{4} g(X, Y)$ which means that $M$ is Einstein. Conversely, by definition $M$ is weakly *Einstein if and only if $\operatorname{Ric}^{*}(X, Y)=\lambda g(X, Y)$. This is equivalent to $R(\Omega)=$ $\lambda \Omega$. Using the frame $\{\alpha, \beta, \bar{\alpha}, \gamma, \delta, \bar{\gamma}\}$, we see that $R(\Omega)=\lambda \Omega$ if and only if $\langle R(\beta), \alpha\rangle=\langle R(\beta), \bar{\alpha}\rangle=\langle R(\beta), \gamma\rangle=\langle R(\beta), \delta\rangle=\langle R(\beta), \bar{\gamma}\rangle=0$. Our assumption that $M$ is an Einstein Hermitian surface implies $W_{2}^{+}=0$ since $W^{+}$is degenerate. Hence we have $\langle R(\beta), \alpha\rangle=\langle R(\beta), \bar{\alpha}\rangle=0$. Furthermore in dimension 4 the Einstein condition is equivalent to $* R=R *$ which implies that $\langle R(\beta), \gamma\rangle=\langle R(\beta), \delta\rangle=\langle R(\beta), \bar{\gamma}\rangle=0$. Hence $M$ is weakly $*$-Einstein and obviously its Ricci tensor is $J$-invariant. This completes the proof of Theorem 1 . The given condition of Corollary 1 implies that $M$ is also a compact Einstein Hermitian surface with constant $s^{*}$ by Theorem 1 . Hence by the well-known result in [4], we can conclude that $M$ is Kähler.

\section{A compact Einstein Hermitian and non-weakly $*$-Einstein manifold of dimension $(4 n+2)$}

The product of odd dimensional spheres $S^{2 n+1} \times S^{2 m+1}$ can be provided with a complex structure [3], defined as follows: let $N_{1}$ and $N_{2}$ be the outward normals to the spheres $S^{2 n+1}$ and $S^{2 m+1}$ sitting inside $\mathbf{C}^{n+1}$ and $\mathbf{C}^{m+1}$, respectively, and let $J_{1}$ and $J_{2}$ be the standard complex structures on these spaces. Since $J_{1} N_{1}$ and $J_{2} N_{2}$ are globally defined vector fields on the respective spheres, we can decompose any vector field $X$ on $S^{2 n+1} \times S^{2 m+1}$ as

$$
X=X_{1}+X_{2}+d_{1}(X) J_{1} N_{1}+d_{2}(X) J_{2} N_{2},
$$

where $X_{1}$ is tangent to $S^{2 n+1}$ and perpendicular to $J_{1} N_{1}$, while $X_{2}$ is tangent to $S^{2 m+1}$ and perpendicular to $J_{2} N_{2}$. The notion of perpendicularity is defined using the standard metrics on $R^{2 n+2}=\mathbf{C}^{n+1}$ and $R^{2 m+2}=\mathbf{C}^{m+1}$, respectively. 
Using this decomposition, we may now define the $(1,1)$-tensor $J_{C}$ by

$$
J_{C} X=J_{1} X_{1}+J_{2} X_{2}-d_{2}(X) J_{1} N_{1}+d_{1}(X) J_{2} N_{2} .
$$

This $J_{C}$ on $S^{2 n+1} \times S^{2 m+1}$ is in fact a complex structure [3]. For each sphere factor in $S^{2 n+1} \times S^{2 m+1}$ we have the Hopf fibration onto a complex projective space and so their product produces a Riemannian submersion $S^{2 n+1} \times S^{2 m+1} \rightarrow$ $C P^{n} \times C P^{m}$. We obtain a decomposition of the tangent space at each point into a horizontal and vertical component. For our purpose we consider the product of odd dimension sphere $S^{2 n+1}$ with itself. Obviously the product metric $g_{\text {prod }}$ of the standard metric on each sphere factor in $S^{2 n+1} \times S^{2 n+1}$ is Einstein. Furthermore, the product metric $g_{\text {prod }}$ on $S^{2 n+1} \times S^{2 n+1}$ is compatible with $J_{C}$. Under the complex structure $J_{C}$, the $*$-Ricci tensor of $g_{\text {prod }}$ is not a functional multiple of the metric $g_{\text {prod }}$. In fact we have $\operatorname{Ric}^{*}(V, V)=0$ and $\operatorname{Ric}^{*}(Y, Y) \neq 0$, where $V$ is a vertical vector and $Y$ is a non-trivial horizontal vector. Summing up the above argument, we can conclude that $M=\left(S^{2 n+1} \times S^{2 n+1}, J_{C}, g_{\text {prod }}\right)$ is a compact Einstein Hermitian manifold which is not weakly $*$-Einstein. This completes the proof of Theorem 2 .

\section{References}

[1] V. Apostolov and P. Gauduchon, The Riemannian Goldberg-Sachs theorem, Internat. J. Math. 8 (1997), no. 4, 421-439.

[2] Ch. Boyer, Conformal duality and compact complex surfaces, Math. Ann. 274 (1986), no. $3,517-526$

[3] E. Calabi and B. Eckmann, A class of compact, complex manifolds which are not algebraic, Ann. of Math. (2) $\mathbf{5 8}$ (1953), 494-500.

[4] J. Kim, On Einstein Hermitian manifolds, Monatsh. Math. 152 (2007), no. 3, 251-254.

[5] _ Rigidity theorems for Einstein-Thorpe metrics, Geom. Dedicata 80 (2000), no. $1-3,281-287$

Department of Mathematics Education Kangwon National University

Chunchon 200-701, Korea

E-mail address: jaeman64@kangwon.ac.kr 